\title{
EQUIDISTRIBUTION OF ORBITS OF ISOMETRIES ON COMPACT RIEMANNIAN MANIFOLDS
}

\author{
ADRIÁN GORDILLO-MERINO AND JOSÉ NAVARRO
}

\begin{abstract}
Let $S$ be a finitely generated semigroup of isometries of a compact Riemannian manifold. We present a simple argument to prove that, if there exists a point $x$ whose orbit $S x$ is dense, then the orbit of any point is equidistributed.
\end{abstract}

\section{Introduction}

The question of the uniform distribution of orbits of groups -or semigroups- of isometries on Riemannian manifolds goes back to $\mathrm{H}$. Weyl, whose theorem ([5]) on the equidistribution on $\mathbb{R} / \mathbb{Z}$ of the arithmetic progressions $\{n \theta\}_{n \in \mathbb{N}}$, for $\theta$ irrational, can be understood as the equidistribution of the orbits of the semigroup generated by the rotation of angle $2 \pi \theta$ radians on the circumference $S_{1}=\mathbb{R} / \mathbb{Z}$.

Some decades later, Arnol'd and Krylov ([1]) used a different notion of equidistribution to study the orbits on a sphere $S_{2}$ of the semigroup generated by two rotations $A, B$. Their result assures that, if there exists a point $x \in S_{2}$ whose orbit is dense, then this orbit is equidistributed, in the sense as follows: consider the $2^{n}$ points obtained by applying exactly $n$ iterations of the two rotations $A$ and $B$ to the point $x$,

$$
A^{n} x, A^{n-1} B x, A^{n-2} B A x, \ldots, B^{n} x ;
$$

then, for any region $\Delta$ (bounded by a piecewise smooth curve), the following equation holds:

$$
\lim _{n \rightarrow \infty} \frac{\text { Number of points among (1.1) inside } \Delta}{2^{n}}=\frac{m(\Delta)}{m\left(S_{2}\right)},
$$

where $m$ is the Lebesgue measure of $S_{2}$.

Recently, Stewart ([4]) has observed that this theorem remains true when considering the orbits of the semigroup generated by a finite number of reflections of $\mathbb{R}^{3}$.

In this short note, we consider the action of a finitely generated semigroup $S$ of isometries on a compact Riemannian manifold $X$. We present a simple argument that characterises the equidistribution of the orbits, in a similar vein to those stated above. To be precise, our Theorem 5 proves that, if there is a dense orbit, then every orbit is equidistributed, in the

Date: June 17, 2019.

2010 Mathematics Subject Classification. Primary 58C35; Secondary 28C99.

Key words and phrases. Equidistribution, isometries, Riemannian manifold.

Both authors have been partially supported by Junta de Extremadura and Fondo Europeo de Desarrollo Regional (GR18001 and IB18087). 
sense that, for any point $x \in X$ and for any system of generators $S=\left\langle a_{0}=1, a_{1}, \ldots, a_{r}\right\rangle$,

$$
\lim _{n \rightarrow \infty} \frac{1}{(r+1)^{n}} \sum_{i_{1}, \ldots, i_{n}=0}^{r} f\left(a_{i_{n}} \ldots a_{i_{1}} x\right)=\frac{1}{m(X)} \int_{X} f d m, \quad \forall f \in \mathcal{C}(X)
$$

where $m$ is the measure induced by a volume form.

As an example, it readily follows that any finitely generated dense subgroup of a compact Lie group is equidistributed (Corollary 7); this proves, in particular, a statement suggested by Stewart in ([4], Remark 6.5).

Finally, let us comment that the notion of equidistribution that we are using in this note is slightly different from those used by the authors mentioned above (see the last Remark of the paper), but it is the crucial point that permits us a simple proof.

\section{EqUidistribution OF ORBITS}

Let $X$ be a compact, metrizable topological space and $\mu$ a measure on the Borel sets in $X$ such that (non-empty) open sets have positive measure and $\mu(X)=1$.

Let $S$ be a finitely generated semigroup acting on $X$ via measure-preserving transformations.

Definition. If we have a certain generating system $S=\left\langle a_{0}=1, a_{1}, \ldots, a_{r}\right\rangle$, then we can define the following operators on the algebra $\mathcal{C}(X)$ of continuous functions on $X$ :

$$
\left(A_{i} f\right)(x)=f\left(a_{i} x\right), \quad(i=0,1, \ldots, r),
$$

and, consequently, the average operator too,

$$
M_{\left\langle a_{0}, \ldots, a_{r}\right\rangle}=\frac{A_{0}+\ldots+A_{r}}{r+1}
$$

as well as its iterations

$$
M_{\left\langle a_{0}, \ldots, a_{r}\right\rangle}^{n}=M_{\left\langle a_{0}, \ldots, a_{r}\right\rangle} \cdot \stackrel{n}{n} \cdot M_{\left\langle a_{0}, \ldots, a_{r}\right\rangle}=\frac{1}{(r+1)^{n}} \sum_{i_{1}, \ldots, i_{n}=0}^{r} A_{i_{n}} \cdot \ldots \cdot A_{i_{1}} .
$$

Thus, for any point $x \in X$,

$$
\left(M_{\left\langle a_{0}, \ldots, a_{r}\right\rangle}^{n} f\right)(x)=\frac{1}{(r+1)^{n}} \sum_{i_{1}, \ldots, i_{n}=0}^{r} f\left(a_{i_{n}} \ldots a_{i_{1}} x\right) .
$$

Definition. The orbit $S x$ of $x \in X$ under the action of $S$ is $\left\langle a_{0}, \ldots, a_{r}\right\rangle$-equidistributed if the following equality holds:

$$
\lim _{n \rightarrow \infty}\left(M_{\left\langle a_{0}, \ldots, a_{r}\right\rangle}^{n} f\right)(x)=\int_{X} f d \mu, \quad \forall f \in \mathcal{C}(X) .
$$

Observe that, if an orbit $S x$ is $\left\langle a_{0}, \ldots, a_{r}\right\rangle$-equidistributed, then $S x$ is dense in $X$.

At a first glance, one could easily disprove the latest definition because of its apparent dependence on the system of generators of the semigroup $S$. Also, it is not clear whether, 
for any two points $x, y$ with the same orbit $(S x=S y)$, the orbit $S x$ is equidistributed if and only if $S y$ is equidistributed. Theorem 1 will provide a situation where both objections are put aside.

Lemma. Let $H_{1}, \ldots, H_{s}$ be linear isometries of a finite-dimensional euclidean vector space $\mathcal{P}$. Then either

$$
\left\|\frac{H_{1}+\ldots+H_{s}}{s}\right\|:=\sup _{\|f\|=1}\left\|\left(\frac{H_{1}+\ldots+H_{s}}{s}\right) f\right\|<1,
$$

or else there exists some unitary vector $f \in \mathcal{P}$, such that:

$$
H_{i} f=H_{j} f, \forall i, j=1, \ldots, s .
$$

Proof: It is clear that $\left\|\frac{H_{1}+\ldots+H_{s}}{s}\right\| \leq 1$. Since the unit sphere of $\mathcal{P}$ is compact, if we suppose that the equality holds, then there exists a unitary vector $f \in \mathcal{P}$ such that

$$
\left\|H_{1} f+\ldots+H_{s} f\right\|=s .
$$

As $\left\|H_{i} f\right\|=1$, for all $i \in\{1, \ldots, s\}$, we easily conclude: $H_{i} f=H_{j} f$.

Theorem 1. Suppose that the algebra of continuous functions on $X$ has a dense (for the uniform convergence topology) subalgebra admitting a decomposition as a sum of certain finite-dimensional subspaces $\mathcal{P}_{l}$, which are all stable under the action of $S$ :

$$
\mathcal{C}(X)=\overline{\sum_{l} \mathcal{P}_{l}}
$$

If there is a dense orbit in $X$, then all orbits under the action of $S$ are $\left\langle a_{0}, \ldots, a_{r}\right\rangle-e q u i$ distributed, for any system of generators $\left\langle a_{0}=1, a_{1}, \ldots, a_{r}\right\rangle$ of $S$.

Proof: First, observe that uniform limits and finite sums of continuous functions on $X$ satisfying equation (2.1) also satisfy it. Besides, note that equation (2.1) is trivial for constant functions.

Consider the subspace $\mathcal{P}_{l}^{0} \subset \mathcal{P}_{l}+\mathbb{R}$ of functions with null integral (where $\mathbb{R}$ is identified with constant functions). Due to the previous considerations, it suffices to prove equation (2.1) for any $f \in \mathcal{P}_{l}^{0}$, and for all $x \in X$, that is:

$$
\lim _{n \rightarrow \infty}\left(M_{\left\langle a_{0}, \ldots, a_{r}\right\rangle}^{n} f\right)(x)=0 .
$$

Consider the following inner product on $\mathcal{P}_{l}^{0}$ :

$$
<f, g>=\int_{X} f g d \mu
$$

which is invariant by $S$, since both the measure $\mu$ is invariant by $S$ and the subspaces $\mathcal{P}_{l}^{0}$ are stable by $S$. Thus, the operators $A_{i}$ are isometries of $\left(\mathcal{P}_{l}^{0},\|\cdot\|\right)$, with $\|\cdot\|$ the corresponding inner product norm, and therefore $\left\|A_{i}\right\|=1$. 
On a finite-dimensional vector space, all separated, locally convex topologies are equivalent, so the pointwise convergence in $\mathcal{P}_{l}^{0}$ stated in (2.2), for each $x \in X$, is equivalent to:

$$
\lim _{n \rightarrow \infty}\left\|M_{\left\langle a_{0}, \ldots, a_{r}\right\rangle}^{n} f\right\|=0 .
$$

If $\left\|M_{\left\langle a_{0}, \ldots, a_{r}\right\rangle}\right\|<1$, we conclude, because $\left\|M_{\left\langle a_{0}, \ldots, a_{r}\right\rangle}^{n} f\right\| \leq\left\|M_{\left\langle a_{0}, \ldots, a_{r}\right\rangle}^{n}\right\|\|f\|$, and $\left\|M_{\left\langle a_{0}, \ldots, a_{r}\right\rangle}^{n}\right\| \leq$ $\left\|M_{\left\langle a_{0}, \ldots, a_{r}\right\rangle}\right\|^{n}$.

Suppose, though, this is not the case. Then, by the previous Lemma, there must exist some null-integral $f \in \mathcal{P}_{l}^{0}$, such that $\|f\|=1$, and, for each $y \in X$,

$$
f\left(a_{i} y\right)=f\left(a_{j} y\right), \forall i, j=0, \ldots, r .
$$

In particular, $f\left(a_{i} y\right)=f\left(a_{0} y\right)=f(y)$, so that $f$ is a constant function on the whole orbit $S x$ of any $x \in X$. By using the hypothesis about the existence of a dense orbit, $f$ must be constant on the whole $X$, thus reaching an absurd conclusion (recall that $f$ is a unitary vector with null integral).

With the same assumptions as in Theorem 1, we obtain the following corollaries:

Corollary 2. An orbit $S x$ is $\left\langle a_{0}=1, a_{1}, \ldots, a_{r}\right\rangle$-equidistributed if and only if it is dense.

Corollary 3. If an orbit $S x$ is $\left\langle a_{0}=1, a_{1}, \ldots, a_{r}\right\rangle$-equidistributed, then, no matter what the choice of the system of generators $\left\langle b_{0}=1, b_{1} \ldots, b_{s}\right\rangle$ of $S$ may be, it is $\left\langle b_{0}, b_{1}, \ldots, b_{s}\right\rangle$-equidistributed.

Besides, if $x, y \in X$ are such that $S x=S y$, then $S x$ is equidistributed if and only if $S y$ is equidistributed.

Corollary 4. If there is an equidistributed orbit, then all the orbits are equidistributed.

\section{ISOMETRIES OF COMPACT RIEMANNIAN MANIFOLDS}

If $X$ is a compact Riemannian manifold, and $\mu=\frac{1}{m(X)} m$, where $m$ is the measure defined by a volume form, then any sub-semigroup $S \subseteq \operatorname{Isom}(X)$ of isometries acts on $(X, \mu)$ via measure-preserving transformations.

Theorem 5. Let $S$ be a finitely generated semigroup of isometries of a compact Riemannian manifold $X$. If there is an orbit under the action of $S$ that is dense, then all the orbits are equidistributed (with respect to any system of generators $\left\langle a_{0}=1, a_{1}, \ldots a_{r}\right\rangle$ of $S$ ).

That is to say, for any point $x \in X$,

$$
\lim _{n \rightarrow \infty} \frac{1}{(r+1)^{n}} \sum_{i_{1}, \ldots, i_{n}=0}^{r} f\left(a_{i_{n}} \ldots a_{i_{1}} x\right)=\int_{X} f d \mu, \quad \forall f \in \mathcal{C}(X) .
$$


Proof: As the Lie group of isometries $\operatorname{Isom}(X)$ is compact ([2]), Mostow's equivariant embedding theorem $([3])$, assures the existence of a finite-dimensional linear representation $\operatorname{Isom}(X) \rightarrow G l(E)$ and an $\operatorname{Isom}(X)$-equivariant closed immersion $X \hookrightarrow E$.

Let $\mathcal{P}_{l}$ be the vector space of continuous functions $f: X \rightarrow \mathbb{R}$ that are restrictions of some polynomial function $\tilde{f}: E \rightarrow \mathbb{R}$ of degree $\leq l$. These vector spaces $\mathcal{P}_{l}$ are stable by the action of $S$, because the isometries of $X$ act by linear transformations on $E$, and the closed immersion $X \hookrightarrow E$ is equivariant.

Moreover, the algebra $\sum_{l \in \mathbb{N}} \mathcal{P}_{l}$ separate points of $X$ so, by the Stone-Weierstrass theorem, it is dense in the algebra of continuous functions on $X$ :

$$
\mathcal{C}(X)=\overline{\sum_{l \in \mathbb{N}} \mathcal{P}_{l}}
$$

and the statement then follows applying Theorem 1.

Corollary 6. Let $S$ be a finitely generated semigroup of linear isometries of the euclidean space $\left(\mathbb{R}^{n+1}, g\right)$. If there exists a point in the unit sphere $S_{n}$ whose orbit is dense, then the orbit of any point of $S_{n}$ is equidistributed in $S_{n}$.

Corollary 7. If $H$ is a dense finitely generated sub-semigroup of a compact Lie group $G$, then it is equidistributed.

In this last statement, the Lie group $G$ is endowed with a left-invariant Riemannian metric, so that the sub-semigroup $H$ acts on that Riemannian manifold by isometries, and the claim is that the orbit of the neutral element $e$ is equidistributed.

Remark. Let $S_{1}=\{z \in \mathbb{C}:|z|=1\}$ be the unit circumference, and let $S=\left\langle a_{0}=1, a_{1}=\right.$ $\left.e^{\theta i}\right\rangle$ be the semigroup generated by $e^{\theta i}$.

If $\frac{\theta}{2 \pi}$ is irrational, then $S$ is dense in $S_{1}$. Hence, Corollary 7 assures that the sequence of points $1, a_{1}, \ldots, a_{1}^{n}, \ldots$ is equidistributed, in the following sense:

$$
\lim _{n \rightarrow \infty} \frac{f(1)+n f\left(a_{1}\right)+\ldots+\left(\begin{array}{l}
n \\
k
\end{array}\right) f\left(a_{1}^{k}\right)+\ldots+f\left(a_{1}^{n}\right)}{2^{n}}=\frac{1}{2 \pi} \int_{0}^{2 \pi} f\left(e^{t i}\right) d t, \quad \forall f \in \mathcal{C}\left(S_{1}\right) .
$$

Acknowledgements. The authors feel deeply indebted to prof. Juan A. Navarro for his generous advice.

\section{REFERENCES}

[1] Arnol'd, V. I., Krylov, A. L.: Uniform distribution of points on a sphere and some ergodic properties of solutions of linear ordinary differential equations in a complex region, Dokl. Akad. Nauk SSSR 148 (1963), 9-12; Engl. transl. Soviet. Math. Dokl. 4 (1963).

[2] Myers, S. B., Steenrod, N. E.: The group of isometries of a Riemannian manifold, Annals of Math., 40 (1939), 400-416. 
[3] Mostow, G. D.: Equivariant embeddings in euclidean space, Annals of Math., 65 (1957), 432-446.

[4] Stewart, I.: Tetrahedral chains and a curious semigroup, Extracta Math., 34 1, (2019) 99-122.

[5] Weyl, H.: Über die Gibbs'sche Erscheinung und verwandte Konvergenzphänomene, Rend. Circ. Matem. Palermo, 330 (1910), 377-407.

Adrián Gordillo-Merino. Departamento de Matemáticas, Universidad de Extremadura, E-06071 BADAJOZ, SPAIN

E-mail address: adgormer@unex.es

José Navarro. Corresponding author. Departamento de Matemáticas, Universidad de Extremadura, E-06071 Badajoz, Spain

E-mail address: navarrogarmendia@unex.es 